\title{
LA EVALUACIÓN DE LAS ACTIVIDADES MUSICALES
}

\author{
Rafael Prieto Alberola \\ Universidad de Alicante
}

\begin{abstract}
RESUMEN. Tras establecer y reflexionar sobre algunos de los condicionamientos que complican las tareas de la evaluación en general y la musical en particular, el autor de este trabajo revisa algunos modelos establecidos y por último sugiere unas pautas aproximadas que faciliten la labor del evaluador de las actividades musicales.
\end{abstract}

ABSTRACT. After establishing and reflecting on some of the conditions which complicate evaluation tasks in general and musical evaluation in particular, the writer of this article revises some of the established models and finally suggests various criteria which facilitate the evaluation of musical activities.

\section{Introducción}

Entre los variados aspectos de la actividad docente, algunas de las tareas que más dudas y contradicciones ocasionan son aquellas relacionadas con la evaluación y con las actuaciones o decisiones asociadas a ella, con independencia del nivel educativo al que hagamos referencia. Además, la sociedad exige un alto índice de calidad en la educación y como consecuencia de ello en la evaluación, considerándola como parte integral del proceso de enseñanza, cuyo fin último es enriquecer la educación del alumno además de informar a la sociedad de la efectividad de un programa. Una adecuada evaluación satisface al maestro, a sus alumnos y familiares y a la sociedad implicada.

La característica social actual de la competitividad se manifiesta en casi todas las áreas de la actividad humana y en la música tiene un significado especial con los concursos y certámenes en donde se pretende elegir al mejor. Esto representa una gran responsabilidad para los jurados y tribunales evaluadores, ya que están en juego, a veces, las ilusiones personales y otras su futuro profesional, con las consiguientes consecuencias económicas, sociales y afectivas. Tal como afirma Saunders, "el maestro debe tener algo más que una opinión subjetiva interior sobre el alumno para situarlo en un grado o en otro" (Saunders citado por Colwell, 1998, p. 93).

\section{La evaluación de las actividades musicales. Características}

La evaluación de las actividades musicales es una de las más complejas por la propia naturaleza de la música. Cada genero artístico está ligado a una determinada dimensión. Por ejemplo, la vida de la obra de arte pictórica tiene la superficie como 
premisa, y se sucede en las dimensiones altura, anchura y en una ilusión de profundidad. En la obra de arte musical es la duración en el tiempo el factor que determina su formato. En el cuadro podemos seguir de la forma más precisa el avance del proceso creativo y descubrir los medios artisticos. Las expresiones artísticas que llegan a nosotros por medio de la vista nos ofrecen sus obras directamente como originales. Aquellas que necesitan del oído como sentido receptor se nos presentan puramente como apuntes, que deben ser realizados por medio de un intermediario, siempre que no queramos asimilarlas en silencio con el puro intelecto o con el oído interno. Por desgracia no siempre puede ser el creador tal intermediario, y especialmente cuando entran en cuestión las múltiples diferencias de saber y de personas en los casos de una masa de intermediarios, se considera esta necesidad como un mal. En la música no podemos dominar los elementos de la creación al mismo tiempo juntos y por separado, como en la pintura, mirando hacia aquí y hacia allá. Lo que hemos oído ha pasado y solamente existe espiritualmente en el recuerdo. Por esta razón, al oyente le será mucho más dificil que a quien mira un cuadro hacerse una idea de obra y estilo.

A la hora de evaluar la composición de un alumno se nos plantea el problema de saber ¿qué hace que una composición sea mala o buena? ya que la composición es un acto subjetivo y la "belleza puede estar en el oído del que escucha". El profesor Bullivant de la Universidad de Oxford (citado por Johnstone 1995), afirmaba a este respecto que "todo lo que podemos decir es esto es lo que hace Bach, o esto Bach lo hace raramente". También es interesante tener en cuenta, como Young establece, que "el valor de una obra de arte depende de cual sea la perspectiva de cada crítico... hay factores que pueden inducir al crítico al error: su capacidad para comprender la obra, el interés que le suscite esa obra y su valoración de la originalidad" (Young, 1997, p. 207). A pesar de ello recientes investigaciones afirman que es posible alcanzar un consenso entre diferentes evaluadores por lo que esta técnica, denominada "técnica de evaluación consensuada" (Hickey, 1999), necesita varios jueces.

La música posee un alto contenido sentimental que fascina a todos los que huyen de pensar o no tienen la voluntad para pensar. Por esta razón, puede ser convertida, a veces, en algo banal y otras veces utilizarse como medio para el embotamiento del pensar. Nietzsche y Thomas Mann se han expresado muy acertadamente sobre esto. También corre el peligro de ser representada por el ejecutante con una orientación únicamente sentimental.

Por tanto, si queremos establecer unos criterios de evaluación sólidos, debemos comenzar por conocer la naturaleza de lo que vamos a evaluar, o sea, la experiencia musical. Filósofos, psicólogos y músicos están de acuerdo en que la música tiene un significado. Sin embargo no todos coinciden a la hora de determinar qué constituye exactamente el significado de la música y mediante que procesos se transmite. Algunos autores como Reimer (1970) y Meyer (1956) sugieren tres categorías a la hora de clasificar estas teorías: Referencialismo, Formalismo y Expresionismo.

Para los referencialistas el valor de una obra de arte se encuentra fuera de ella y hay que buscar en las ideas, emociones, actitudes y situaciones a las que la música se refiere. En este sentido la función de la música es evocar o recordar alguna de las referencias extramusicales. Reimer utiliza la teoría artística Ilamada "Realismo socialista" como un buen ejemplo de Referencialismo en donde el arte está considerado como un servidor de las necesidades políticas. La función de la música, en este ejemplo, es la de apoyar las necesidades del Estado. Lo importante es el mensaje que puede ser intelectual, político, emocional, etc. Desde la época de Platón y 
en otras más recientes como la de Tolstoy, se ha pensado que las obras de arte son capaces de hacer aflorar emociones no artísticas y que este hecho ayuda a conducir a las masas en la dirección que le interesa al poder establecido. Cooke (1959) al igual que Tolstoy ve la música como "lenguaje" en cuanto a que ciertos intervalos poseen un significado preciso. Teoría que no comparte Langer (1953) cuando afirma que "la música, aparte de algunos temas onomatopéyicos como el sonido del cu-co o la campana, no tiene un significado literal".

Los formalistas establecen que el significado de la música radica en sus "cualidades formales". "La experiencia artística para el formalista es fundamentalmente intelectual" (Reimer, 1970, p. 56). Los formalistas aceptan que muchas obras de arte contienen referencias del mundo fuera de la obra de arte pero las consideran "totalmente irrelevantes" al significado de ésta. La visión extremada del formalismo musical está representada por Stravinsky, "la música es por naturaleza debil para expresar algo y si esto ocurre entonces es una ilusión y no una realidad" (Stravinsky citado por Cooke, 1959).

Para los expresionistas el significado de la obra de arte tiene dos aspectos, el relativo a la obra de arte en sí y a la relación entre la obra de arte, el compositor y el oyente. La combinación de los dos se ha llamado "Expresionismo absoluto", cuyo eclecticismo parece lo más apropiado, desde nuestro punto de vista, para entender el significado de la música. La actitud del evaluador musical en relación a estas teorías puede ser determinante para su trabajo. La belleza puede ser muy subjetiva, sin embargo, siguiendo a Hickey (1996), creemos que es posible establecer una serie de criterios objetivos que nos permitan valorar la actividad musical de forma fundamentada.

Aunque dada la actualidad del tema hay bastante bibliografía que nos puede ayudar en cuanto al tratamiento de la evaluación en general (Pérez Juste, 1995; Stufflebeam, 1988), a la hora de centrarnos en la evaluación de las actividades musicales nos encontramos con la escasez de referencias. El informe del Chief State School Officers afirma que "pocos o ningún profesor (en formación) recibe mucha preparación en evaluación" (Colwell, 1998, p. 79). Los profesores de música, tampoco han contribuído, al menos en forma de publicaciones, a facilitar a sus alumnos y colegas el encuentro con esta gran "asignatura pendiente" como es la evaluación de las actividades musicales.

El hecho de que en los planes de estudios de los conservatorios se preste escasa o ninguna atención a la preparación pedagógica de sus alumnos nos parece inadecuado, especialmente porque al terminar los largos estudios musicales obtienen un titulo de profesor o profesor superior, el cual les permite como salida profesional más plausible la de la enseñanza. En una encuesta realizada en el Conservatorio norteamericano de Nueva Inglaterra se vió que nunca se habían impartido clases o cursos de evaluación. En 1991 Stiggins afirmó que incluso algunos alumnos que habían seguido un curso de evaluación se consideraban "deficientemente preparados" (Stiggins citado por Collins 1998, p. 105).

Coincidimos con la National Association of Schools of Music cuando defiende la idea de que si eres un buen músico puedes ser un buen profesor, pero nos parece necesario completar esta opinión diciendo que para ser un buen profesor de música hacen falta algunas características que el hecho magnífico de ser un buen músico no garantiza necesariamente.

Aunque es un tema que lleva preocupando mucho a los educadores musicales desde hace décadas, ha sido gracias a la publicación de los National Standards para 
la Educación Musical en USA, que los profesionales de la educación musical han puesto manos a la obra reflexionando de forma escrita en publicaciones sobre este particular. En 1991 el norteamericano Leonhard, presidente de MENC (Music Education National Conference), afirmaba que la evaluación musical solo se realizaba en asignaturas como la Historia o la Teoría de la Música y en cuanto a la interpretación se valoraba en terminos de "asistencia", "actitud" "cooperación" (conceptos extramusicales) y como mucho, el profesor evaluaba si el estudiante "se equivocaba de nota" o "no afinaba" (MENC 1995, p. 16). La mayoría de las referencias encontradas tienen su origen en el mundo anglosajón, siendo adecuado constatar que el mayor porcentaje de trabajos están dedicados a la educación musical en primaria o secundaria y muy raramente a otros tipos de colectivos como son los estudiantes de magisterio y los profesores de música en activo. En parte porque los colegios de primaria y secundaria conforman el mayor colectivo y también puede ser porque, como explica Donald Taebel citado por Collins (1998), "la evaluación de profesores es azarosa y compleja, quizás porque los conceptos de enseñanza y evaluación son multifacéticos" (p. 76).

En cualquier caso, estamos de acuerdo en que, como el expresidente de MENC afirma, "los criterios de evaluación intensificarán su importancia en los próximos años y serán muy importantes en la música porque muchos administradores de escuela y aquellos que tienen que tomar las decisiones tienen poca idea de cómo evaluar el aprendizaje de la música de una manera válida" (MENC 1995, p. 36).

\section{Hacia una metodología de evaluación de las actividades musicales}

El objetivo de este trabajo es reflexionar sobre la evaluación de las actividades musicales y sugerir unas pautas metodológicas a nivel de evaluación que faciliten la labor del evaluador musical y colabore a reducir la complejidad del problema.

Para este fin nos parece oportuno comenzar por la revisión de algunos documentos como la "Ley Orgánica de Ordenación General del Sistema Educativo" española de 1990 y la "Education Reform Act" inglesa de 1988. Estas dos leyes fueron el origen del Decreto 20/1992 de 17 de febrero de 1992 de la Comunidad Valenciana y del "National Curriculum" inglés para la educación musical, respectivamente, publicado el mismo año. En el caso inglés, el informe del "Task Group on Assessment and Testing" (TGAT) clarifica la relación entre enseñanza y aprendizaje al enfatizar que "el proceso evaluativo...debería ser una parte integral del proceso educativo, proporcionando contínuamente tanto un "feedback" como un "feedforward". (Es decir hacia el alumno y hacia el profesor). (TGAT, A Report paragraph 4). Aunque no se planificaron exámenes a nivel nacional, si se establecieron los llamados "End of key stage descriptions" para utilizarlos en la evaluación sumativa y los "Attainement targets" en los que se indican las destrezas, el conocimiento y la comprensión que se espera que el alumno posea al final de la etapa. La mayoría de los criterios son esencialmente de carácter cuantitativo, por ejemplo el Key Stage 2 para la actividad de interpretar (performing), dice que los niños deberán interpretar "signos más complejos" mientras que en el Key stage 3 dice que deberán interpretar "variedad de signos".

El gran educador musical inglés Swanwick critica alguna de las redacciones del National Curriculum como cuando en éste se lee que se pedirá al alumno "un amplio rango de ideas". Lo cual Swanwick considera incorrecto explicando que si se valoraran de esta forma algunos de los preludios de Bach, construídos sobre una 
o dos ideas, no se les podría, según este criterio, otorgar una buena nota (Swanwick 1997 , p. 207). Este mismo autor defiende que cualquier forma de evaluación depende de la evidencia de cambios cualitativos. En esta línea constructivista, se manifiestan autores como Coll al afirmar que "la distinción entre estas actividades de enseñanza y aprendizaje y las actividades de evaluación pierde en gran parte su sentido". (Coll, 1994, p. 182).

En cuanto al Curriculum para la educación musical en primaria de la Comunidad Valenciana, se establecen 15 criterios de evaluación con explicación de cada uno de ellos. Es por tanto más explícito que el inglés aunque el valenciano se refiere a toda el area artística en general. En la primavera de 1999, el Departamento de Educación norteamericano publicó, a través del National Assessment of Educational Progress (NAEP), un informe sobre evaluación realizada en 1995 y 1997 a nivel nacional. El marco de la evaluación musical consta de: Contenidos y procesos. Los contenidos incluyen: 1. Conocimiento y comprensión de la música. 2. Destrezas perceptivas, tecnicas, expresivas e intelectuales. Los procesos incluyen: 1. Crear. 2. Interpretar y 3. Responder (NAEP, 1999).

Este modelo está basado en la promulgación de los National Standards for Music Education, los cuales tienen algunos críticos como aquellos que dicen que es imposible valorar una composición realizada por un alumno que es incapaz de leer o recordar lo que ha escrito. Deborah Haimo dice, refiriéndose a los Standards, que son "poco concisos, vagos y se prestan a ser malinterpretados por el estudiante" (Haimo citada por Colwell 1998:86). Sin embargo muchos otros, como Diane Ravitch, los defienden diciendo que "el propósito de los Standards es señalar a los alumnos y profesores lo que se puede hacer esforzándose" (Ravitch citada por Colwell 1998, p. 86). El Secretario de Educación norteamericano Richard Riley dice que los objetivos más importantes que los Standards pretenden conseguir, son extramusicales como "aprender a resolver problemas, pensar creativamente y desarrollar disciplina mental" (National Standards.. 1994).

En Inglaterra Keith Swanwick ha popularizado en el último cuarto de siglo su modelo Ilamado CAP (Composition, Audition, Performance) reforzando la opinión de los que defienden que el alumno de música debe realizar actividades en estos tres campos de forma paralela, desde el comienzo de los estudios para adquirir una completa educación musical. En este sentido, nosotros basamos nuestra sugerencia metodológica atendiendo a estos tres campos fundamentales de la educación musical.

Antes de elegir un modelo de evaluación conviene considerar muchos factores: el tipo de alumnos, el tiempo y los medios de que disponemos, el contenido y el proceso de la enseñanza, la evaluación de este proceso y los resultados o evaluación del producto. Tambien debemos asegurarnos de que el método que utilizamos mida aquello para lo cual fue diseñado. Tal como establece Lines, citada por Cope (1996) la evaluación musical debe tener 4 atributos: 1. Proceso contínuo y dinámico en donde el estudiante es ayudado a aprender de su propia experiencia. 2. Multidimensional: utiliza medidas formales e informales. 3. Colaborativo entre alumnos y profesor y 4. Auténtico es decir que contenga tareas que tengan su aplicación en la vida real (Lines citada por Cope, 1996, p. 67).

"La evaluación debe ser objetiva buscando cambios en el comportamiento como por ejemplo la actitud de confianza en sus propios valores musicales y el 
incremento de su pasión por la música" (Colwell, 1998, p. 87). El objetivo a evaluar debe ser claro y explícito. En lugar de decir: "el alumno debe cantar con musicalidad" nos parece más concreto decir: "la frase debe ser cantada con el uso apropiado de la respiración, dinámica, tempo, tensión y relajación". Definir claramente, con consistencia y objetividad, lo que se va a evaluar así como los criterios y coeficientes de evaluación.

Hay que evaluar solo lo que se ha explicado en clase. Utilizar sistemas de anotación de las observaciones que no interrumpan la dinámica del aula. Es mejor evaluar diferentes aspectos de la actividad del alumno que no contentarse con dar una nota global a toda su actuación. Evaluar actividades que sean significativas para el alumno, es decir, que tengan su aplicación práctica en situaciones de la "vida real".

El método que utilicemos puede ser susceptible de mejora, pero debemos asegurarnos de que se utilice exactamente igual para cada alumno. Que los resultados que se obtengan sean los mismos aunque fuera otro el evaluador. Cualquier sistema de evaluación fallará si las tareas son demasiado difíciles para el nivel, si el alumno no interpreta bien los enunciados de las preguntas o si es muy costoso el procedimiento de evaluación.

Con el fin de que nuestro trabajo consiga resultados fiables es necesario realizar las evaluaciones con regularidad, secuenciando los objetivos intermedios hasta conseguir el objetivo final del curso. Las correciones deben ser en lo posible individualizadas para lo cual es bueno hacer tocar "solos" en el aula, ya que sirve para observar el progreso individual y además ayuda a reducir la ansiedad que produce un concierto público.

El Arts Propel patrocinado por la Fundación Rockefeller y el Havard's Project Zero (citados por Hickey 1999), sugieren que los maestros tienen que evaluar la evaluación realizada por el estudiante en base a estos criterios: Que sean "específicas", que estén "relacionadas con el problema", y que aporten "sugerencias de mejora". Esto es especialmente adecuado y necesario en el caso de los profesores de las facultades de educación.

Una forma de llevar a la práctica la evaluación, después de tener en cuenta todo lo anterior, es :

Imaginar los dos extremos de una escala graduada. Organizar estos criterios en una "línea de calidad" y a continuación describir puntos intermedios. Utilizar terminos constructivos y positivos aún en los más bajos niveles de la escala para favorecer la autoestima y la motivación del estudiante.

De acuerdo con Hickey (1999), los criterios generales son: estética, creatividad y coherencia en la cumplimentación del trabajo. La Encyclopedia of Assessment Dimensions editada por el Harvard's Project Zero dice que "los criterios de evaluación ofrecen el medio más honesto y auténtico de medir la calidad del trabajo creativo de los niños en un area que es muy subjetiva". "Los criterios de evaluación hacen que ésta sea concreta y objetiva, proporcionan feed-back al alumno y desarrollan su capacidad crítica" (Hickey, 1999, p. 34).

Existen muchas clasificaciones de tipos de evaluación. De todas las revisadas la que más se acerca a nuestro ideal, sobre metodología de evaluación, es la realizada por Goolsby, el cual establece cuatro tipos: Inicial, Sumativa, Diagnóstica y Formativa (Goolsby, 1999, p. 57). 


\section{Tipos de instrumentos de evaluación musical.}

Entre las mas utilizadas podemos señalar las escalas graduadas:

Para construir una escala graduada hay que tener en cuenta: la edad y el nivel del alumno, la naturaleza de la actividad a evaluar, el tipo de tarea y su dimensión específica (Saunders citado por Cope, 1996, p. 83). Estas escalas pueden ser "contínuas" si la consecución de un item da por supuesto el dominio del anterior o "aditivas" en las que los items son independientes. Si son consensuadas y asumidas por los alumnos, éstos se responsabilizarán más de las tareas.

Actualmente se están utilizando algunas alternativas que simplemente citaremos aquí para no extendernos y porque todvía están en fase de experimentación: Los "portfolios" que son continentes de evidencia (grabaciones, redacciones, composiciones, etc.). Existen varios tipos: "Product portfolio", "Program portfolio" y "Process portfolio". La mayor ventaja consiste en que en ellos, se concede igual importancia al proceso que al producto, que el progreso del estudiante se puede observar y reflejar de forma individualizada y que fomenta el trabajo colaborativo entre los alumnos. Su desventaja tal vez sea la excesiva categorización del trabajo del estudiante y que además requiere mucho tiempo de dedicación del profesor, además de su coste. En 1994 Colwell mostró que el coste por estudiante fue de 37 dólares. Otros instrumentos alternativos pueden ser las entrevistas, los trabajos de clase y "los diarios, los cuales facilitan la comunicación y la relación entre profesor y alumno" (Robinson, 1995, p. 86).

Salvo en el caso de evaluadores muy expertos, las actividades de interpretación deberían ser grabadas en cinta o video (Carlin, 1996) para que posteriormente puedan ser valoradas por cada item independiente. De esta manera, también tendría el maestro y el alumno una referencia objetiva sobre la que reflexionar a la hora de evaluar y no hacerlo de memoria, lo que puede dar lugar a desacuerdos evitables. Igualmente en las actividades de audición, los criterios de evaluación expuestos están indicados para música grabada ya que hay items que solo son valorables cuando se dispone de una grabación para poder ser escuchada repetidas veces.

Colwell nos brinda su experimentada comprensión cuando dice que "si es difícil evaluar el arte, más dificil es evaluar la creatividad y la improvisación" (Colwell, 1998, p. 88). Sin embargo al referirnos a la evaluación de la composición tal como establece Hickey, "los maestros no tienen obligación de considerar a la composición como una actividad a evaluar cuantitativamente" (Hickey, 1999, p.87). Quizás aquí podrían ser muy útiles los métodos cualitativos o la mezcla de las dos metodologías. El uso de los "portfolios" puede encontrar aquí una buena oportunidad para su utilización. También nos puede ser muy útil prestar atención a los trabajos de composición para observar el progreso realizado en materias como la teoría de la música, la coherencia formal, el uso de conceptos del lenguaje musical, etc.

En la "evaluación basada en la interpretación" (performance-based assessment), que es un nuevo nombre para algo antiguo, se usan escalas del 1 al 5 o de la A a la F, etc. El problema es que las distancias entre cada uno de los grados no son idénticas. Al final, según establece Robinson (1995), se corre el peligro de caer en lo subjetivo.

En síntesis, se trata de ser capaces de identificar los comportamientos musicalmente adecuados y medir lo que esperamos que los alumnos aprendan utilizando diferentes medios de evaluación: "Multiple-measures approach". Es importante hacer 
participar a los alumnos en el diseño e implementación de la evaluación y también en la comunicación de los resultados, de esta manera fomentaremos su nivel de motivación y de responsabilidad.

\section{Modelo que proponemos}

Basándonos en el modelo establecido por Swanwick (1979) CAP (Composition, Audition and Performance, son las actividades que este autor considera imprescindibles en la formación musical, de ahí que establezca este modelo), nuestra sugerencia parte de que el alumno sea evaluado en estas tres actividades a cualquier nivel. Si nos ceñimos a nuestro trabajo en la formación del profesorado especialista en educación musical para primaria, las actividades de evaluación en este ámbito educativo incluirán también las tres citadas. Se presenta la actividad y después los objetivos que se pretenden con cada actividad a los que cada profesor debería, si desea seguir este modelo, asignar una escala graduada de 4 puntos en los que establecería las diferentes posibilidades de nivel de realización. Ejemplo: A: Consigue todo, B: Consigue más de la mitad, C: Consigue la mitad, D: Consigue menos de la mitad. A cada uno de estos niveles el profesor otorgará un coeficiente de evaluación que puede ser: D: de 0 a 2'5, C: de 2'6 a 5, B: de 5'1 a 7'5, A: de 7'6 a 10. Como ejemplo proponemos las actividades y objetivos a evaluar en cada una de ellas.

\section{CREATIVIDAD:}

Actividad: Componer a partir de una letra dada, una obra musical instrumentada para la escuela cuya forma musical será: Introducción, A A' B A" y Coda que constará de 32 compases, asignando 8 a cada frase sin contar la Introducción y la Coda que en este contexto deben ser breves, es decir, no más de 4 compases cada una. Variar, improvisar y transportar la frase A. Presentar y evaluar su trabajo y el de un compañero.

1. Adecuación de la acentuación prosódica a la musical y de la música a la letra: modalidad elegida y carácter expresivo incluyendo matices y articulaciones. Los criterios, reducidos a la acentuación, los aplicamos a cada frase de 8 compases.

2. Facilidad de entonación. Preferencia por los grados conjuntos, utilizando algunos saltos fáciles: 3as, o 4as. o 5as, cuando éstas se dan entre los grados I y IV o I y V. Dentro de la tesitura adecuada.

3. Construcción lógica: Fraseo coherente.

4. Interés didáctico (que se practique un concepto de los contenidos que se estan estudiando en ese momento) y/o musical.

5. Armonía adecuada (en donde todas las notas melódicas se puedan justificar) utilizando sólo acordes de Tónica, Dominante y Subdominate.

6. Distribución de las voces para una plantilla de voz, flauta dulce, carillón, metalófono y xilófono, mas uno de afinación indeterminada como por ejemplo el pandero. El objetivo será la facilidad de ejecución y el interés de cada una de las partes.

7. Transportar la melodía para un instrumento transpositor. El objetivo consistirá en la corrección de las notas y su tesitura.

8. Realizar variaciones sobre la frase A de la melodía escrita. 
9. Improvisar sobre la base armónica establecida en el no 5 para la frase A. El objetivo será la correción de las notas y el interés estructural y melódico.

10. La evaluación crítica y la presentación correcta del trabajo.

\section{INTERPRETACIÓN:}

Actividad: Interpretar "a solo" formando parte de un grupo instrumental y/o vocal y dirigir una obra seleccionada para la escuela primaria. Evaluar la propia interpretación y la de un compañero o grupo.

1. Análisis formal de la partitura a interpretar atendiendo a fraseo, tonaliadad, modulaciones si las hay y cadencias. Por último, señalar las células características.

2. Establecimiento y defensa de las decisiones de interpretación (dirección) en base al análisis realizado: tempo, matices, articulación, detección de problemas y forma de resolverlos.

3. Interpretación: Afinación. 4: Ajuste rítmico y tempo. 5: Matices. 6: Articulación. 7: Carácter expresivo. 8: Sonido.

9. Actitud como interprete ante la partitura y ante la audiencia.

10. Selección de partituras y evaluación de la interpretación propia y de la de sus compañeros.

\section{AUDICIÓN:}

Actividad: Seleccionar una obra musical para la escuela primaria, utilizándola para actividades de expresión corporal y de audición crítica.

1. Actividades de movimiento corporal sugeridas por la música que se escucha.

2. Comentarios acerca de las sensaciones personales experimentadas al escuchar una música concreta.

3. Evaluación crítica sobre la selección de la obra en referencia al objetivo: audiencia, valor didáctico, etc.

4. Análisis crítico sobre la obra seleccionada en base a su forma y estructura.

5. Evaluación crítica sobre la instrumentación utilizada.

6. Análisis de la armonía utilizada.

7. Evaluación crítica de los fragmentos improvisados. Adecuación armónica e interés melódico.

8. Carácterísticas estilísticas explicando porqué y en qué momentos de la obra se realzan.

9. Situar la obra en su contexto sociocultural.

10. Repetir cantando, tocando un instrumento o escribiendo en notación tradicional las células características.

\section{Conclusiones}

Parece que está fuera de duda la necesidad de asegurar unas evaluaciones de calidad. Creemos oportuno partir de la base sincera y humilde de que la justicia completa a la hora de evaluar es una utopía. Todo diseño de evaluación como todo producto 
humano es susceptible de mejora y por tanto sujeto a crítica. Pensamos que la mejor forma de aproximarse al objetivo de realizar evaluaciones justas es precisamente reconociendo nuestra incapacidad para ser evaluadores perfectos. De esta manera, poniendo nuestro empeño en hacerlo de la mejor manera que seamos capaces, estaremos alerta para cometer los menos fallos posibles y dispuestos a la crítica para cambiar con afanes de mejora. Tal como afirma Colwell "El conocimiento de nuestras virtudes y defectos como evaluadores es la autovía hacia la mejora" (Colwell, 1998, p. 82).

Enseñar y aprender en la escuela, y especialmente en la de música, son tareas complejas. Saber lo que se ha aprendido dentro o fuera del colegio es todavía más complicado, además "el tiempo y el esfuerzo que se necesitan, a veces pueden llevar a la conclusión de que no compensa" (Colwell, 1998, p. 85). La habilidad para evaluar se consigue realizando prácticas desde el primer año de la carrera de magisterio, permitiendo que el alumno experimente con ideas y técnicas no usuales y con estudiantes de diferentes edades, habilidades y actitudes.

Cada una de las actividades que hemos expuesto, posee una particularidad a la hora de ser evaluadas, por ejemplo la interpretación es compleja de evaluar porque ocurren muchas cosas al mismo tiempo: afinación, ajuste rítmico, matices, articulación, expresividad, etc. Para esta actividad en particular puede ser muy apropiada la utilización del video, que permitirá al profesor y/o al alumno revisar tantas veces como necesite la interpretación, para poder evaluarla de manera más objetiva que confiando todo a la memoria.

Los coeficientes de evaluación variarán dependiendo del peso que cada maestro considere darle a cada item. En cualquier caso, sea cual sea la decisión que adopte el maestro, sí queremos enfatizar nuestra absoluta convicción de que los coeficientes de evaluación deben estar resueltos a partir de una reflexión personal y desde luego, antes de comenzar la evaluación, escritos, publicados (a ser posible consensuados con los alumnos) y por supuesto, para ser respetados fielmente.

Una de las desventajas de este modelo que sugerimos radica en los coeficientes de evaluación asignados a cada item y a cada uno de los cuatro niveles de valoración de cada uno de ellos, ya que como afirma Robinson (1995) "la distancia entre los distintos niveles no es a menudo igual". Creemos que la forma de paliar este problema es que el evaluador clarifique sus objetivos y los priorice con el fin de poder asignar un coeficiente numérico lo más aproximado posible a sus convicciones que aunque subjetivas estén meditadas y fundamentadas. Nuestro modelo sólo pretende ser una sugerencia para que a partir de la cual, cada maestro cambie, mejore y adapte a su pensamiento y contexto lo que le sea útil.

No se trata de que el evaluador que empieza a familiarizarse con estas tareas lo haga todo desde el primer dia, es mejor experimentar con técnicas diferentes para encontrar la que mejor va a cada situación y cuándo es mejor utilizarlas. Siempre procurando comenzar con pequeñas actividades de evaluación piloto de poco coste, que nos sirvan para tomar decisiones de mejora. Como afirma Collins, "enseñando lo enseñable y midiendo lo medible los maestros facilitarán una buena relación del estudiante con la música" (Collins, 1998, p. 76).

Para finalizar nos parece oportuno recordar dos pensamientos de dos grandes educadores. Uno de ellos, Goolsby, defiende la idea de cambiar la actitud tradicional del evaluador, es decir, en lugar de "buscar y detectar el error", se debería intentar "buscar y detectar el aprendizaje". En noviembre de 1998 otro gran edu- 
cador como es Leonhard, citado por Colwell (1998), dijo que "la evaluación debería basarse en el principal propósito del arte: favorecer el conocimiento y el amor por el arte" (p. 84).

\section{Referencias bibliográficas}

ASMUS, E. P. (1999). Music assessment concepts. Music educators journal. Vol. 86.

BRASHER. E. (et al.) (1999). Managing assessment in music education. Teaching music. Vol. 7.

CARLIN, J. (1996). Videotape as an assessment tool. Teaching music. Vol. 3.

COLL, C. (1994). El compromiso con la Reforma. Cuadernos de Pedagogía. Vol. 91.

COLLINS, I. H. (1998). Assessment and evaluation in music teacher education. Arts education policy review. Vol. 98.

COLWELL, R. (1998). Preparing student teachers in assessment. Arts education policy review. Vol. 99.

COLWELL, 6. (1999). The 1997 assessment in music: red flags in the sunset. Arts education policy review. Vol. 100.

COOKE, D. (1959). The languages of music. London: OUP.

COPE, C. O. (1996). Steps towards effective assessment. Music educators journal. Vol. 83.

Decreto 20/1992 de 17 de febrero de la Comunidad Valenciana. DOGV.

GOOLSBY, T. W. (1995). Portfolio assessment for better evaluation. Music educators journal. Vol. 82.

GOOLSBY, T. W. (1999). Assessment in instrumental music. Music educators journal. Vol. 86.

HICKEY, M. (1996) Consensual Assessment of Children's Musical Compositions. (Research project presentation, New York State School Music Association Convention). New York.

HICKEY, M. (1999). Assessment rubrics for music compositions. Music educators journal. Vol. 85.

JOHNSTONE, A. (1995). Tecniques of Composition in the British and Irish University Curriculum. British Journal of Music Education. V.12. Cambridge Press.

LANGER, S. (1953). Feeling and form. Routledge: London.

LOGSE. (1990).

MENC (Music educators national conference). (1995). Teaching music. Vol. 2.

MEYER, L. B. (1956). Emotion and meaning in music. University of Chicago Press.

NATIONAL CURRICULUM for England and Wales. (1991).

NATIONAL STANDARDS FOR ARTS EDUCATION. (1994). 
(NAEP) NATIONAL ASSESSMENT OF EDUCATIONAL PROGRESS. (1999) Department of Education. USA.

PÉREZ JUSTE, R. (1995). Evaluación de programas educativos. En Medina Rivilla, A. y Villar Angulo, L. M. (Cood.). Evaluación de programas educativos, centros y profesores. Ed. Universitas S.A.: Madrid.

REIMER, B. (1970). A philosophy of music education. New Jersey: Prentice Hall.

ROBINSON, M. (1995). Alternative assessment tecniques for teachers. Music educators journal. Vol. 83.

STAUFFER, S. L. (1999). Beginning assessment in elementary general music. Music educators journal. Vol. 86.

STUFFLEBEAM, D. L. y SHANKFIELD, A. J. (1987). Evaluación sistemática. Guía teórica y práctica. Ed. Paidós-MEC. Barcelona.

STUFFLEBEAM, D. L. (1988). Normas para evaluaciones. En Pascual, R. (Cood). La gestión educativa ante la innovación y el cambio. Ed. Narce. Madrid.

SWANWICK, K. (1979). A basis for Music Education. Windsor: Nfer-Nelson.

SWANWICK, K. (1997). Assessing musical quality in the National Curriculum. British journal of music education. 14:3. 205-215.

(TGAT) TASK GROUP ON ASSESSMENT AND TESTING. (1990). Department of Education. England.

YOUNG, J. O. (1997). Relativism and the evaluation of art. Journal of aesthetic education. Vol. 31. 